\title{
纳米氧化锰负载钛基电催化膜制备及其苯甲醇催化氧化性能
}

\author{
田文杰1,2 王 虹 ${ }^{1,2}$ 尹 振 ${ }^{1,3, *}$ 杨 映 ${ }^{1,2}$ 李建新 ${ }^{1,2, *}$ \\ ('天津工业大学, 分离膜与膜过程省部共建国家重点实验室, 天津 $300387 ;{ }^{2}$ 天津工业大学 材料科学与工程 \\ 学院, 天津 $300387 ;{ }^{3}$ 天津工业大学, 环境与化学工程学院, 天津 300387)
}

\begin{abstract}
摘要：利用乳液法制备出 $\mathrm{MnO}_{x}$ 纳米颗粒, 将其负载于微孔管式钛膜制得 $\mathrm{MnO}_{x}$ 负载钛基电催化膜 $\left(\mathrm{MnO}_{x} / \mathrm{Ti}\right)$. 运用X射线衍射 $(X R D) 、 X$ 射线光电子能谱(XPS)、扫描电子显微镜(SEM)、循环伏安法 (CV)和计时电流法 $(\mathrm{CA})$ 等表征方法系统考察了不同焙烧温度下 $\mathrm{MnO}_{x}$ 晶型结构、 $\mathrm{MnO}_{x} / \mathrm{Ti}$ 催化膜电化学性能以及催化氧化苯甲醇 的变化规律. 结果表明: 随着焙烧温度的升高, $\mathrm{MnO}_{x}$ 的晶型由初始的Birnessite- $\mathrm{MnO}_{2}$ 逐渐转变为 $\mathrm{K}_{0.27} \mathrm{MnO}_{2}$, 再 由 $\mathrm{Mn}_{3} \mathrm{O}_{4}$ 最终转变为 $\alpha-\mathrm{MnO}_{2}$. 所得 $\mathrm{MnO}_{x} / \mathrm{Ti}$ 膜中, $\alpha-\mathrm{MnO}_{2}$ 晶粒尺寸小于 $30 \mathrm{~nm}$, 结晶度较高, 颗粒分布均匀. 同 时, 由于其含有不饱和配位的锰原子和氧空位以及与基体 $\mathrm{Ti}$ 之间存在键合作用, 表现出优异的电化学性能和催 化性能. 以 $450^{\circ} \mathrm{C}$ 焙烧所得的 $\alpha-\mathrm{MnO}_{2} / \mathrm{Ti}$ 为阳极构建电催化膜反应器催化氧化苯甲醇. 在反应温度为 $25^{\circ} \mathrm{C}, 50$ $\mathrm{mmol} \cdot \mathrm{L}^{-1}$ 苯甲醇水溶液, 电流密度为 $2 \mathrm{~mA} \cdot \mathrm{cm}^{-2}$, 停留时间为 $15 \mathrm{~min}$ 的条件下, 膜反应器苯甲醇转化率达 $64 \%$, 苯甲醛选择性为 $79 \%$.
\end{abstract}

关键词: 多孔钣膜; 电催化膜反应器; 锰氧化物; 苯甲醇; 催化氧化

中图分类号: O643; O646; TQO53

\section{Preparation of Nano-Manganite Loaded Titanium Electocatalytic Membrane for the Catalytic Oxidation of Benzyl Alcohol}

\author{
TIAN Wen-Jie ${ }^{1,2} \quad$ WANG Hong ${ }^{1,2} \quad$ YIN Zhen ${ }^{1,3, *} \quad$ YANG Ying ${ }^{1,2} \quad$ LI Jian-Xin ${ }^{1,2, *}$ \\ ( ${ }^{1}$ State Key Laboratory of Separation Membranes and Membrane Processes, Tianjin Polytechnic University, Tianjin 300387, \\ P. R. China; ${ }^{2}$ School of Materials Science and Engineering, Tianjin Polytechnic University, Tianjin 300387, P. R. China; \\ ${ }^{3}$ School of Environmental and Chemical Engineering, Tianjin Polytechnic University, Tianjin 300387, P. R. China)
}

\begin{abstract}
MnO}_{x}$ nanoparticles obtained by the emulsion method were loaded on a microporous tubular titanium membrane to prepare a functional $\mathrm{MnO}_{x} / \mathrm{Ti}$ electrocatalytic membrane. The effects of calcination temperature on the crystal structure of $\mathrm{MnO}_{x}$ as well as the electrochemical properties and catalytic performance to oxidize benzyl alcohol of $\mathrm{MnO}_{x} / \mathrm{Ti}$ membrane were systematically investigated by $\mathrm{X}$-ray diffraction (XRD), X-ray photoelectron spectroscopy (XPS), scanning electron microscopy (SEM), cyclic voltammetry (CV), chronoamperometry (CA), and other characterization methods. The results indicated that the crystal structure of $\mathrm{MnO}_{x}$ was gradually transformed from Birnessite- $\mathrm{MnO}_{2}$ to $\mathrm{K}_{0.27} \mathrm{MnO}_{2}$, and finally to $\alpha$ $\mathrm{MnO}_{2}$ from $\mathrm{Mn}_{3} \mathrm{O}_{4}$ with increasing calcination temperature. The $\alpha-\mathrm{MnO}_{2}$ particles in the $\mathrm{MnO}_{x} / \mathrm{Ti}$ electrocatalytic membrane showed high crystallinity and uniform particle size (less than $30 \mathrm{~nm}$ ). The superior electrochemical properties and catalytic performance of $\alpha-\mathrm{MnO}_{2} / \mathrm{Ti}$ membrane obtained at a calcination temperature of $450{ }^{\circ} \mathrm{C}$ could be attributed to the binding effects between unsaturated coordination atoms of $\mathrm{Mn}$ and oxygen
\end{abstract}

Received: January 20, 2015; Revised: June 15, 2015; Published on Web: June 17, 2015.

"Corresponding authors. YIN Zhen, Email: yinzhen@tjpu.edu.cn. LI Jian-Xin, Email: jxli@tjpu.edu.cn. Tel: +86-22-83955798.

The project was supported by the National Natural Science Foundation of China $(21206119,21303119)$ and Changjiang Scholars and Innovative Research Team in University of Ministry of Education of China (IRT13084).

国家自然科学基金(21206119, 21303119)和长江学者和创新团队发展计划(IRT13084)项目资助

(C) Editorial office of Acta Physico-Chimica Sinica 
vacancies with the $\mathrm{Ti}$ substrate. The $\alpha-\mathrm{MnO}_{2} / \mathrm{Ti}$ membrane obtained at $450{ }^{\circ} \mathrm{C}$ was used as the anode to assemble an electrocatalytic membrane reactor to oxidize benzyl alcohol. $64 \%$ conversion of benzyl alcohol and $79 \%$ selectivity to benzaldehyde was achieved under the operating conditions: reaction temperature 25 ${ }^{\circ} \mathrm{C}$, aqueous benzyl alcohol solution of $50 \mathrm{mmol} \mathrm{L}{ }^{-1}$, current density $2 \mathrm{~mA} \cdot \mathrm{cm}^{-2}$, and residence time $15 \mathrm{~min}$.

Key Words: Porous titanium membrane; Electrocatalytic membrane reactor; $\quad$ Manganite; Benzyl alcohol; Catalytic oxidation

\section{1 引言}

探索环境友好且具有经济价值的烃类化合物 选择性氧化体系是当前有机合成工业以及催化领 域的重点研究方向之一, 其中醇类的选择性氧化因 其工业应用广泛而备受关注. ${ }^{1}$ 苯甲醇催化氧化是醇 类选择性氧化的典型反应, 目前的催化剂体系研究 主要集中于负载型纳米贵金属、 ${ }^{2}$ 杂多酸、 ${ }^{3}$ 过渡 金属氧化物4等. 纳米贵金属虽有较高的催化活性, 但存在成本高、易流失、分离回收困难等问题. ${ }^{5}$ 杂 多酸催化剂的化学性质同时具有酸性和氧化性, 兼 具有“液相”的特质, 虽具有优良的催化性能, 但存在 比表面积小、利用率较低、产物难以从反应体系 中分离等问题. ${ }^{6}$ 而过渡金属氧化物因其热稳定性 好、抗中毒性能优异、易产生氧空位、选择氧化 反应选择性高且成本较低等诸多优点在多相催化 氧化反应中成为研究重点. ${ }^{7.8}$ 作为典型的过渡金属 氧化物, 锰氧化物具有储量丰富、价格低廉、环境 友好、多变的晶体类型、多种微观隧道结构以及 可变的价态等优势, ${ }^{9}$ 被越来越多地应用于催化氧 化. $\mathrm{Su}$ 等 ${ }^{10}$ 对比了 $\alpha-\mathrm{MnO}_{2} 、 \mathrm{MnO} 、 \mathrm{Mn}_{2} \mathrm{O}_{3} 、 \mathrm{Mn}_{3} \mathrm{O}_{4}$ 、 $\mathrm{V}_{2} \mathrm{O}_{5} 、 \mathrm{CuO} 、 \mathrm{Co}_{2} \mathrm{O}_{3} 、 \mathrm{NiO} 、 \mathrm{Fe}_{2} \mathrm{O}_{3}$ 等催化剂选择性 氧化苯甲醇制备苯甲酫的催化效果, 结果表明, $\alpha$ $\mathrm{MnO}_{2}$ 的催化活性较高, 苯甲醇的转化率为 $37.7 \%$, 选 择性为 $98.6 \%$. 锰氧化物虽然具有较好的催化性能, 但是由于反应条件比较苛刻, 需要添加大量氧化剂 等诸多问题而限制其应用.

本研究小组 ${ }^{11-14}$ 曾报道构建具有催化-强化传质 一体化协同作用的电催化膜反应器, 用于电催化氧 化正丙醇、四氟丙醇和葡萄糖等低碳链醇类物质. 这种反应器以负载纳米 $\mathrm{MnO}_{2}$ 的导电微孔钛膜 $\left(\mathrm{MnO}_{x} /\right.$ $\mathrm{Ti}$ )为阳极, 不锈钢网为阴极, 外接蠕动洜提供恒定 负压. 通过优化反应参数, 丙醇转化率可达 $99 \%$, 丙 酸的选择性为 $80 \%$; $^{11,12}$ 四氟丙醇的转化率达 $92 \%$, 四 氟丙酸钠的选择性大于 $99 \%{ }^{13}$ 葡萄糖的转化率高达 $99 \%$, 葡萄糖及葡萄糖二酸的选择性达到 $99 \% .{ }^{14}$ 其
膜反应器的高效性关键在于两个方面: 一方面通过 电解产生水产生的· $\mathrm{OH}$ 自由基引发锰的价态发生连 续变化, 即电子的转移与锰的价态变化相互结合进 而实现催化氧化的目的. 另一方面通过膜反应器强 化传质促进产物在膜电极表面传递与及时分离, 从 而提高膜电极的催化效能. ${ }^{15}$ 然而, $\mathrm{MnO}_{x} / \mathrm{Ti}$ 催化膜制 备过程采用的是硝酸锰热分解法, 制备过程中存在 氮氧化物的释放会造成环境污染.

本文利用乳液法制备出 $\mathrm{MnO}_{x}$ 纳米颗粒, 将其负 载于微孔管式钛膜制得 $\mathrm{MnO}_{x}$ 负载钛基电催化膜 $\left(\mathrm{MnO}_{x} / \mathrm{Ti}\right)$, 系统考察了不同的焙烧温度下 $\mathrm{MnO}_{x}$ 晶 型结构、催化膜的形貌、化学组成、电化学性能 以及催化氧化苯甲醇的变化规律.

\section{2 实验部分}

\section{1 实验材料及仪器}

材料: 油酸、 $\mathrm{KMnO}_{4}$ 、苯甲醇、苯甲醛、苯甲 酸、无水乙醇均为分析纯, 天津光复化学试剂; 微 孔钛膜, 上海一鸣过滤有限公司.

仪器: 可调式直流稳压电源(M8811, 南京美尔 诺电子有限公司); 蠕动泵(BT100L, 保定雷弗流体 科技有限公司), 真空管式烧结炉(XMT-S9000, 天津 中环实验电炉有限公司); 高速离心机(TG-20, 长沙 英泰仪器有限公司); 压录仪(US61M/IV-9500, 北京 中西科技); 气相色谱仪(Agilent GC-7890, 色谱柱 DB-FFAP: $30 \mathrm{~m} \times 0.25 \mathrm{~mm} \times 0.25 \mu \mathrm{m}$, 美国 $)$; $X$ 射线 衍射仪(Bruker AXS D8, Cu $K_{\alpha} \lambda=0.15406 \mathrm{~nm}$, 德国); 场发射扫描电子显微镜(Hitach S-4800, 日本); 电化 学工作站(Zahner-Zennium, 德国); X射线光电子能 谱仪(Thermofisher K-Alpha, 英国).

\section{$2.2 \mathrm{MnO}_{x}$ 和 $\mathrm{MnO}_{x} / \mathrm{Ti}$ 电催化膜的制备}

$10 \mathrm{~mL}$ 油酸与 $10 \mathrm{~mL}$ 无水乙醇混合均匀后, 将 10 $\mathrm{mL}$ 浓度为 $0.2 \mathrm{~mol} \cdot \mathrm{L}^{-1}$ 的 $\mathrm{KMnO}_{4}$ 溶液逐滴缓慢滴加 到混合溶液中, 形成黑色乳液. 乳液以 $9000 \mathrm{r} \cdot \mathrm{min}^{-1}$ 的速率离心 $10 \mathrm{~min}$, 得到 $\mathrm{MnO}_{x}\left(25^{\circ} \mathrm{C}\right)$. 产物置于管 
式炉中, 空气气氛下以 $5^{\circ} \mathrm{C} \cdot \mathrm{min}^{-1}$ 的升温速率分别加 热至250、350、 $450{ }^{\circ} \mathrm{C}$, 保温 $3 \mathrm{~h}$, 自然冷却至室温.

原始钛膜的预处理采用 $80^{\circ} \mathrm{C} \mathrm{NaOH}$ 碱洗 $15 \mathrm{~min}$, $20 \%$ 草酸溶液超声浸泡 $1 \mathrm{~h}$, 蒸馏水洗涤、晾干后浸 渍于上述 $\mathrm{MnO}_{x}$ 乳液 $1 \mathrm{~h}, 80^{\circ} \mathrm{C}$ 烘干, 得到 $\mathrm{MnO}_{x} / \mathrm{Ti}(25$ $\left.{ }^{\circ} \mathrm{C}\right)$. 将其置于管式炉中, 空气气氛下以 $5^{\circ} \mathrm{C} \cdot \mathrm{min}^{-1}$ 的 升温速率分别加热至250、350、450 ${ }^{\circ} \mathrm{C}$, 保温 $3 \mathrm{~h}$, 自然冷却至室温.

影响 $\mathrm{MnO}_{x}$ 以及 $\mathrm{MnO}_{x} / \mathrm{Ti}$ 的晶型结构的因素包括 焙烧温度、升温速率、保温时间等. 为考察焙烧温 度的影响, 本实验将升温速率固定为 $5{ }^{\circ} \mathrm{C} \cdot \mathrm{min}^{-1}$, 保 温时间为 $3 \mathrm{~h}$.

\section{$2.3 \mathrm{MnO}_{x} / \mathrm{Ti}$ 电化学性能测试}

采取三电极体系, 以电催化膜为工作电极, 饱 和甘录电极为参比电极, 铂片电极为辅助电极(铂) 尺寸为 $15 \mathrm{~mm} \times 15 \mathrm{~mm} \times 0.1 \mathrm{~mm}), 0.1 \mathrm{~mol} \cdot \mathrm{L}^{-1}$ 硫酸 钠电解质溶液, 采用德国Zahner-Zennium电化学工 作站进行循环伏安测试和计时电流测试. 其中, 循 环伏安测试电压范围为 $-1.5-1.5 \mathrm{~V}$, 扫描速率50 $\mathrm{mV} \cdot \mathrm{s}^{-1}$; 计时电流运行电压为 $1 \mathrm{~V}$, 运行时间为 $1 \mathrm{~h}$.

\section{$2.4 \mathrm{MnO}_{x} / \mathrm{Ti}$ 电催化膜反应器的组装}

电催化膜反应器的组装与 $\mathrm{Li}$ 等 ${ }^{12}$ 所报道相似, 如 图1所示. 以 $\mathrm{MnO}_{x} / \mathrm{Ti}$ 电催化膜为阳极, 不锈钢网为 阴极, 分别通过导线与直流稳压电源相连, 极间距 约为 $25 \mathrm{~mm}$, 采用死端过滤, 电催化膜外接蠕动洜提 供分离负压, 促进溶液由膜外流入膜内.

\section{5 膜反应器实验参数}

停留时间 $t_{\mathrm{R}}(\mathrm{min})$ 是指反应物由膜外表面通过孔 道到达膜内表面所经历的时间, 通过改变蠕动原的 体积流速调控停留时间, 计算方法是膜孔体积 $V\left(\mathrm{~cm}^{3}\right)$

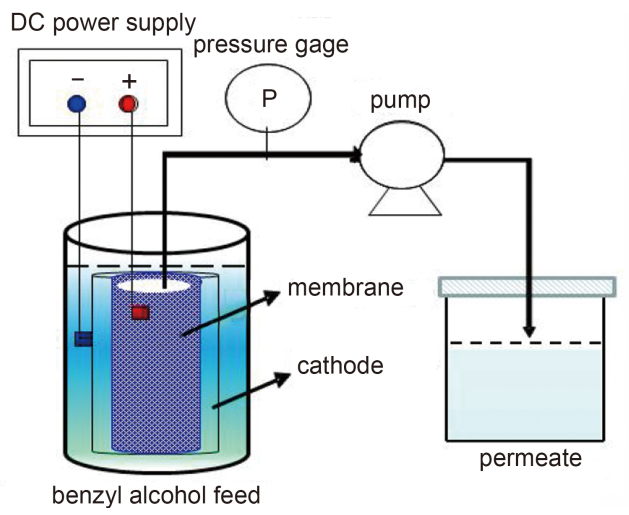

图1 $\mathrm{MnO}_{x} / \mathrm{Ti}$ 电催化膜反应器装置图

Fig.1 Scheme of $\mathrm{MnO}_{x} / \mathrm{Ti}$ electrocatalystic membrane reactor
和孔隙率 $P$ 的乘积与溶液体积流速 $v\left(\mathrm{~mL} \cdot \mathrm{min}^{-1}\right)$ 的比 值.

$$
t_{\mathrm{R}}=(V \times P) / v
$$

电流密度 $J\left(\mathrm{~mA} \cdot \mathrm{cm}^{-2}\right)$ 是指单位时间内通过膜表 面的电流强度, 计算方法为总电流 $I(\mathrm{~mA})$ 与膜表面 积 $A\left(\mathrm{~cm}^{2}\right)$ 的比值.

$$
J=I / A
$$

\section{6 苯甲醇氧化反应}

配制 $50 \mathrm{mmol} \cdot \mathrm{L}^{-1}$ 苯甲醇溶液, 以 $0.1 \mathrm{~mol} \cdot \mathrm{L}^{-1}$ 硝 酸钠为电解质, 设置停留时间为 $15 \mathrm{~min}$, 电流密度为 $2 \mathrm{~mA} \cdot \mathrm{cm}^{-2}$. 苯甲醇氧化反应过程见图2 所示.

\section{7 苯甲醇、苯甲醛的检测}

利用气相色谱检测原料液和透过液中苯甲 醇、苯甲醛的含量. 检测条件如下: 进样口温度 260 ${ }^{\circ} \mathrm{C}$, 氢火焰离子化检测器(FID) $280{ }^{\circ} \mathrm{C}$, 氢气流速 40 $\mathrm{mL} \cdot \mathrm{min}^{-1}$, 进样量 $1 \mu \mathrm{L}$, 分流比 $1: 25$. 柱温箱升温程 序为初温 $120^{\circ} \mathrm{C}$, 以 $25^{\circ} \mathrm{C} \cdot \mathrm{min}^{-1}$ 的升温速率升温至 $210{ }^{\circ} \mathrm{C}$ 保温 $5 \mathrm{~min}$, 继续以 $25^{\circ} \mathrm{C} \cdot \mathrm{min}^{-1}$ 的升温速率升 温至 $240{ }^{\circ} \mathrm{C}$ 保持 5 min.

\section{3 结果与讨论}

\section{1 $\mathrm{MnO}_{x}$ 催化剂的表征}

通过XRD表征不同焙烧温度下 $\mathrm{MnO}_{x}$ 的晶型结 构, 结果见图3. 由图3可知, 随着焙烧温度的升高, 其衍射峰逐渐增强, 表明 $\mathrm{MnO}_{x}$ 结晶度逐渐提高. 此 外, 在 $25^{\circ} \mathrm{C}$ 时 $\mathrm{MnO}_{x}$ 衍射峰较宽, 表明其存在非晶结 构, 在 $11.0^{\circ} 、 36.0^{\circ} 、 65.0^{\circ}$ 出现Birnessite- $\mathrm{MnO}_{2}$ 特征 衍射峰. ${ }^{16}$ 由图3还可以发现, $250{ }^{\circ} \mathrm{C}$ 所得到 $\mathrm{MnO}_{x}$ 晶 型为 $\mathrm{K}_{0.27} \mathrm{MnO}_{2}$ 和 $\mathrm{Mn}_{3} \mathrm{O}_{4}$ 的混合物. 其中, $12.4^{\circ}$ 、 $42.8^{\circ}$ 为 $\mathrm{K}_{0.27} \mathrm{MnO}_{2}$ (JCPDS NO.52-0556) 的特征衍射峰 . $^{17}$ 在 $18.0^{\circ} 、 28.8^{\circ} 、 31.1^{\circ} 、 32.2^{\circ} 、 36.0^{\circ} 、 59.8^{\circ}$ 的衍射 峰为 $\mathrm{Mn}_{3} \mathrm{O}_{4}$ (JCPDS NO.24-0734)的特征衍射峰. ${ }^{18}$ 而 当温度升至 $350{ }^{\circ} \mathrm{C}$, 在 $12.65^{\circ} 、 17.9^{\circ} 、 28.8^{\circ}$ 、 $37.5^{\circ} 、 41.9^{\circ} 、 49.8^{\circ} 、 56.3^{\circ} 、 60.2^{\circ} 、 69.6^{\circ}$ 等处为 $\alpha-$ $\mathrm{MnO}_{2}$ 衍射峰(JCPDS NO.44-0141)..$^{19}$ 继续升温至 450 ${ }^{\circ} \mathrm{C}, \mathrm{Mn}_{3} \mathrm{O}_{4}$ 全部转变为 $\alpha-\mathrm{MnO}_{2}$ 结构(图3). $\mathrm{MnO}_{x}$ 负载

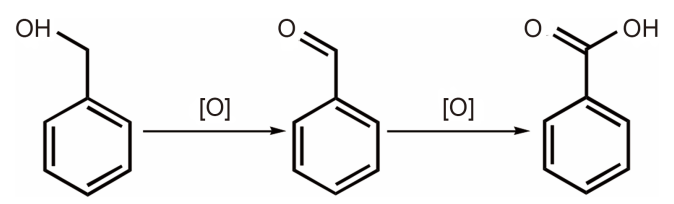

图2 苯甲醇氧化反应示意图

Fig.2 Sketch map of benzyl alcohol oxidation reaction 


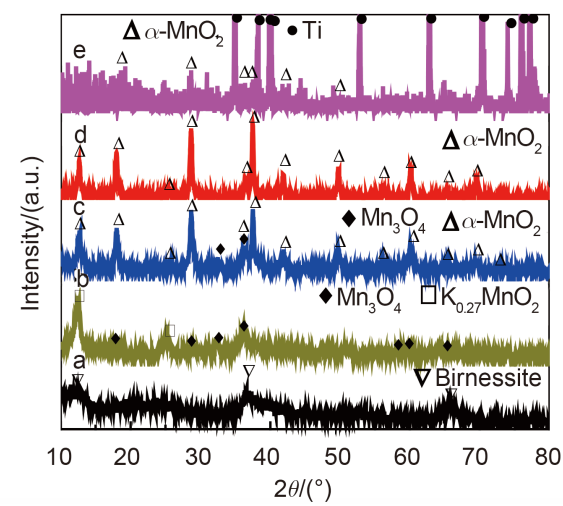

图3 不同焙烧温度的 $\mathrm{MnO}_{x}$ 及 $450{ }^{\circ} \mathrm{C} \mathrm{MnO}_{x} / \mathrm{Ti}$ 电催化膜的 XRD谱图

Fig.3 XRD spectra of $\mathrm{MnO}_{x}$ calcined at different calcination temperatures and $\mathrm{MnO}_{x} / \mathrm{Ti}$ electrocatalystic membrane calcined at $450{ }^{\circ} \mathrm{C}$

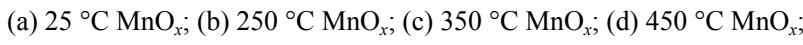
(e) $450{ }^{\circ} \mathrm{C} \mathrm{MnO}_{x} / \mathrm{Ti}$

于基膜, 在 $450{ }^{\circ} \mathrm{C}$ 焙烧温度下, $\mathrm{MnO}_{x} / \mathrm{Ti}$ 膜出现 $\alpha-$ $\mathrm{MnO}_{2}$ 特征衍射峰.

总之, 通过XRD的表征和分析可以得出焙烧过 程中 $\mathrm{MnO}_{x}$ 的晶型转变过程: 由Birnessite- $\mathrm{MnO}_{2}$ 首先 转变为 $\mathrm{K}_{0.27} \mathrm{MnO}_{2}$, 尔后经过 $\mathrm{Mn}_{3} \mathrm{O}_{4}$, 最终变成 $\alpha-\mathrm{MnO}_{2}$.

此外, 晶粒尺寸是催化剂的另一重要参数. 结 合XRD谱图, 根据谢乐公式 $D=K \gamma \cdot(B \cos \theta)^{-1}(K$ 为 Scherrer常数, $D$ 为晶粒垂直于晶面方向的平均厚度,
$B$ 为样品衍射峰半高宽, $\theta$ 为衍射角), 可以计算得出 $\mathrm{MnO}_{x}$ 的晶粒尺寸, 结果如表1所示. 由表1可知, 随着 焙烧温度的升高, 由于晶型转变过程中成核和再生 长, $\mathrm{MnO}_{x}$ 晶粒尺寸逐渐增大. 比如, $25{ }^{\circ} \mathrm{C}$ 条件下 Birnessite- $\mathrm{MnO}_{2}$ 的晶粒尺寸为 $2.5 \mathrm{~nm}, 250{ }^{\circ} \mathrm{C}$ $\mathrm{Mn}_{3} \mathrm{O}_{4}$ 晶粒尺寸为 $6.3 \mathrm{~nm}, \mathrm{~K}_{0.27} \mathrm{MnO}_{2}$ 的尺寸为 12.3 nm. $350{ }^{\circ} \mathrm{C} \mathrm{Mn}_{3} \mathrm{O}_{4}$ 的晶粒尺寸增加至 $19.02 \mathrm{~nm}, \alpha$ $\mathrm{MnO}_{2}$ 的晶粒尺寸为 $19.2 \mathrm{~nm} .450{ }^{\circ} \mathrm{C}$ 所制备的 $\alpha-$ $\mathrm{MnO}_{2}$ 晶粒尺寸最大, 达 $28.9 \mathrm{~nm}$.

\section{2 $\mathrm{MnO}_{x} / \mathrm{Ti}$ 的表征}

$\mathrm{MnO}_{x}$ 作为催化剂, 首先以乳液形式涂覆于基体 表面, 之后经过焙烧去除油酸等纳米颗粒的表面有 机物. 利用扫描电镜考察焙烧温度对于 $\mathrm{MnO}_{x}$ 在基体 表面形貌、粒径的影响, 结果如图4所示.

表1 谢乐公式计算 $M_{n} \mathrm{O}_{x}$ 晶粒尺寸

Table 1 Crystallite dimension of $\mathrm{MnO}_{x}$ calculated by Scherrer equation

\begin{tabular}{ccc}
\hline Crystal structure of $\mathrm{MnO}_{x}$ & $T /{ }^{\circ} \mathrm{C}$ & Crystallite dimension $/ \mathrm{nm}$ \\
\hline Birnessite- $\mathrm{MnO}_{2}$ & 25 & 2.5 \\
$\mathrm{Mn}_{3} \mathrm{O}_{4}$ & 250 & 6.3 \\
$\mathrm{~K}_{0.27} \mathrm{MnO}_{2}$ & 250 & 12.3 \\
$\mathrm{Mn}_{3} \mathrm{O}_{4}$ & 350 & 19.0 \\
$\alpha-\mathrm{MnO}_{2}$ & 350 & 19.2 \\
$\alpha-\mathrm{MnO}_{2}$ & 450 & 28.9 \\
\hline
\end{tabular}
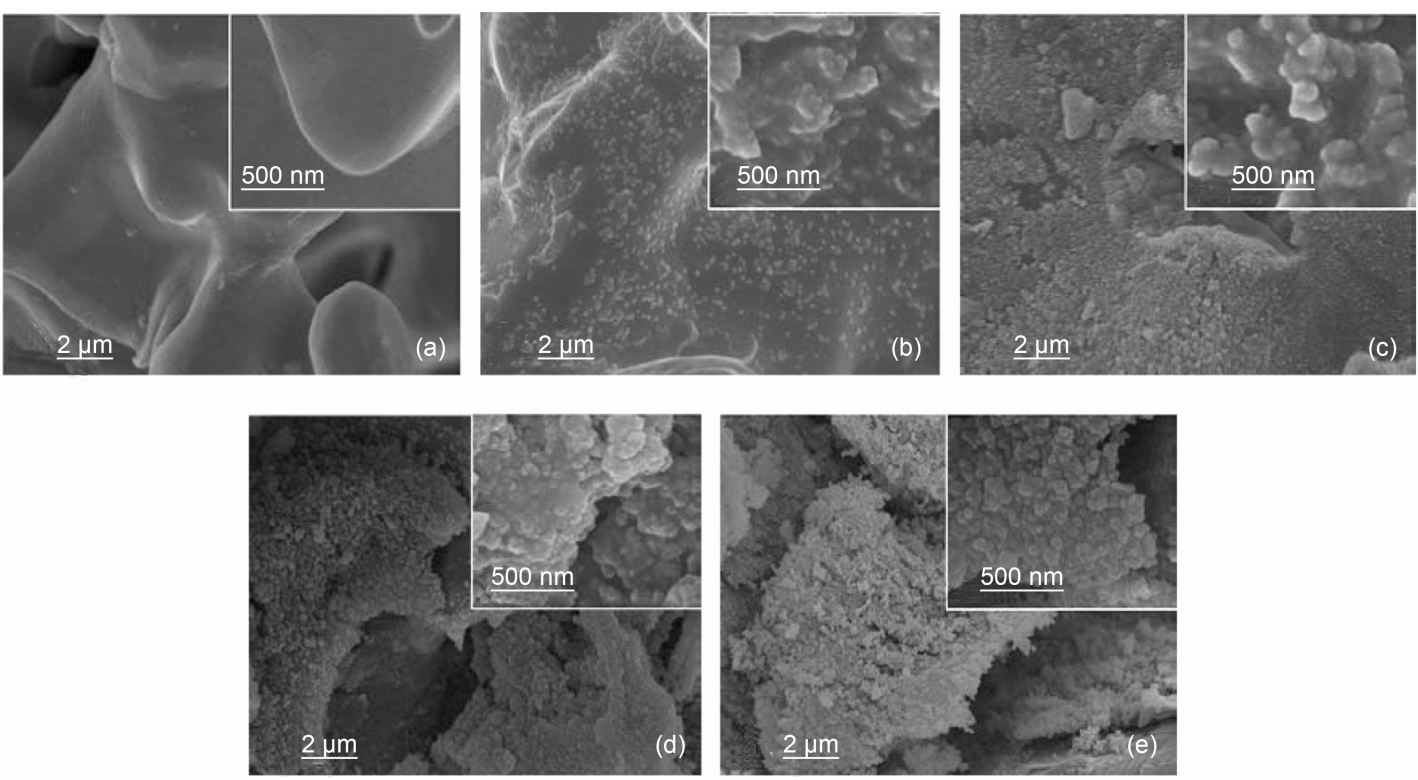

图4 不同焙烧温度 $\mathrm{MnO}_{x} / \mathrm{Ti}$ 电催化膜扫描电镜图片

Fig.4 SEM images of $\mathrm{MnO}_{x} / \mathrm{Ti}$ electrocatalystic membrane calcined at different calcination temperatures (a) Ti matrix; (b) $25^{\circ} \mathrm{C}$; (c) $250{ }^{\circ} \mathrm{C}$; (d) $350{ }^{\circ} \mathrm{C}$; (e) $450{ }^{\circ} \mathrm{C}$ 
由图4发现: 预处理后钛基膜表面光滑, 无明显 颗粒物; 而 $\mathrm{MnO}_{x} / \mathrm{Ti}$ 膜表面存在明显的颗粒物, 且随 着焙烧温度提高, 颗粒物明显增多, 粒径更加均匀, 且颗粒在基膜表面的覆盖也更加均匀. 压录法测量 膜比表面积, 结果显示其在热处理前后无明显变化.

为考察 $\mathrm{MnO}_{x}$ 涂层在基膜表面的化学组成、价 态以及与基膜的相互作用, 采用X射线光电子能谱 分别对 $\mathrm{MnO}_{x} / \mathrm{Ti}$ 膜表面 $\mathrm{Mn}$ 、 $\mathrm{Ti}$ 的结合能进行分析, 结果见图5.

如图5(a)是Mn 2p 的XPS谱图, 由 $\mathrm{Mn}^{2+} 、 \mathrm{Mn}^{3+}$ 、 $\mathrm{Mn}^{4+}$ 的响应信号共同叠加构成, 分别以 $1 、 2 、 3$ 来表 示. 通过拟合得出 $\mathrm{MnO}_{x} / \mathrm{Ti}$ 电催化膜表面 $\mathrm{Mn}$ 的化学 状态.$^{20,21}$ 由图5(a)可以看出, 钛膜负载催化剂后, $25^{\circ} \mathrm{C}$ $\mathrm{MnO}_{x} / \mathrm{Ti}$ 有 $\mathrm{Mn} 2 p$ 轨道的谱峰出现, 且锰的价态主要 为 $\mathrm{Mn}^{2+} 、 \mathrm{Mn}^{3+}$. 随着焙烧温度升高, $\mathrm{Mn}^{4+}$ 的含量明显 增大, $\mathrm{Mn}$ 的结合能增加, 即锰的价态逐渐升高. 450 ${ }^{\circ} \mathrm{C}$ 所得的 $\alpha-\mathrm{MnO}_{2} / \mathrm{Ti}$ 中, $\mathrm{Mn}^{2+}$ 所占比重显著降低, 锰 的价态主要为 $\mathrm{Mn}^{3+} 、 \mathrm{Mn}^{4+}$, 表示 $\alpha-\mathrm{MnO}_{2}$ 表面仍存在 不同价态的锰原子.

为了考察 $\mathrm{MnO}_{x}$ 的负载对基膜的影响, 对 $\mathrm{Ti}$ 的化

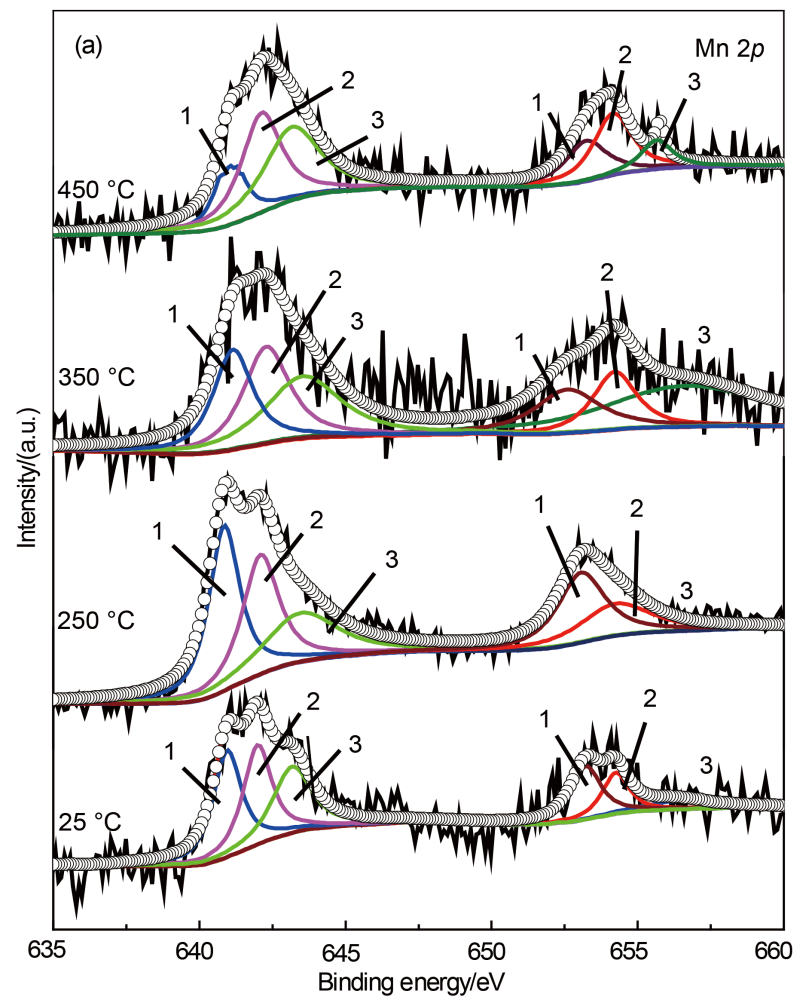

学结合能的变化进行分析, 如图 5(b)所示. 由图 5 (b) 可知, 25-350 ${ }^{\circ} \mathrm{C}$ 所得 $\mathrm{MnO}_{x} / \mathrm{Ti}$ 电催化膜中 $\mathrm{Ti}$ $2 p$ 的结合能与基膜相比未出现大的变化. 然而, 当 热处理温度升至 $450{ }^{\circ} \mathrm{C}$, 所制备的 $\alpha-\mathrm{MnO}_{2} / \mathrm{Ti}$ 膜中 $\mathrm{Ti}$ 结合能显著降低, 与原始钛膜相差 $0.54 \mathrm{eV}$. 由此 可以推断 $\alpha-\mathrm{MnO}_{2}$ 与基体 $\mathrm{Ti}$ 的之间存在强烈的相互 作用, 形成了 Mn-O-Ti键合. 由此, 改变了 Ti所处的 化学环境.

为进一步分析 $\alpha-\mathrm{MnO}_{2} / \mathrm{Ti}$ 电催化膜表面的锰氧 化物与基体之间的相互作用, 对比了 $450{ }^{\circ} \mathrm{C}$ 所得 $\alpha-$ $\mathrm{MnO}_{2} / \mathrm{Ti}$ 膜材料、 $\alpha-\mathrm{MnO}_{2}$ 粉末以及基体钛膜中的 $\mathrm{O}$ $1 s 、 \mathrm{Mn} 2 p 、 \mathrm{Ti} 2 p$ 轨道XPS 谱图, 结果如图6所示. 由图6中O谱峰可以发现: $\mathrm{Ti}$ 基膜经过预处理, 表面 的杂质以及氧化物减少, $\mathrm{O}$ 元素主要以 $\mathrm{OH}^{-}$形式存 在. $\alpha-\mathrm{MnO}_{2}$ 粉末主要为晶格氧 $(\mathrm{Mn}-\mathrm{O}-\mathrm{Mn})$. 而 $\alpha-$ $\mathrm{MnO}_{2} / \mathrm{Ti}$ 催化膜表面 $\mathrm{O}$ 以晶格氧( O $1 s$ metal)、吸附 氧 $\left(\mathrm{O} 1 s \mathrm{OH}^{-}\right)$和结合水 $\left(\mathrm{H}_{2} \mathrm{O}\right)$ 三种形式存在. ${ }^{22}$ 其中, 吸附氧含量显著高于 $\alpha-\mathrm{MnO}_{2}$ 粉末, 表明 $\alpha-\mathrm{MnO}_{2} / \mathrm{Ti}$ 晶格中 $\mathrm{Mn}-\mathrm{O}$ 键较弱, 存在不饱和配位的锰原子以 及氧空位, 即具有更多的催化活性中心. ${ }^{23}$

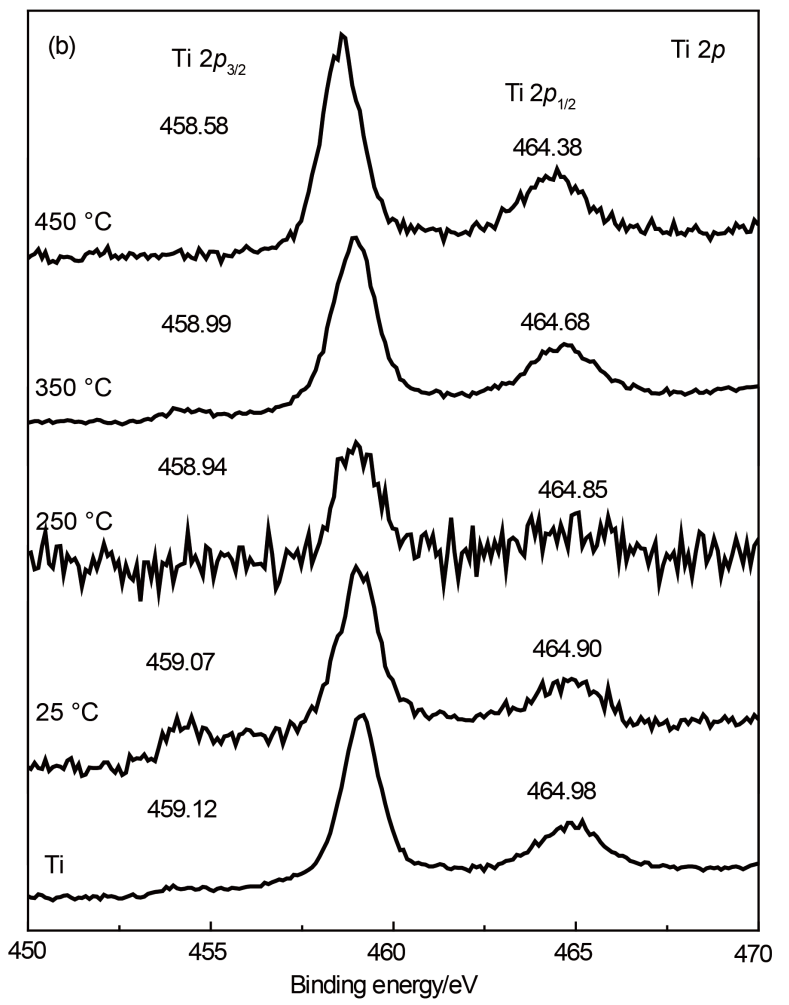

图5 $\mathrm{MnO}_{x} / \mathrm{Ti}$ 电催化膜XPS Mn $2 p$ 和Ti $2 p$ 谱图

Fig.5 XPS spectra of Mn $2 p$ and Ti $2 p$ orbits obtained from $\mathrm{MnO}_{x} /$ Ti electrocatalytic membrane (1) $\mathrm{Mn}^{2+} ;(2) \mathrm{Mn}^{3+} ;(3) \mathrm{Mn}^{4+}$ 

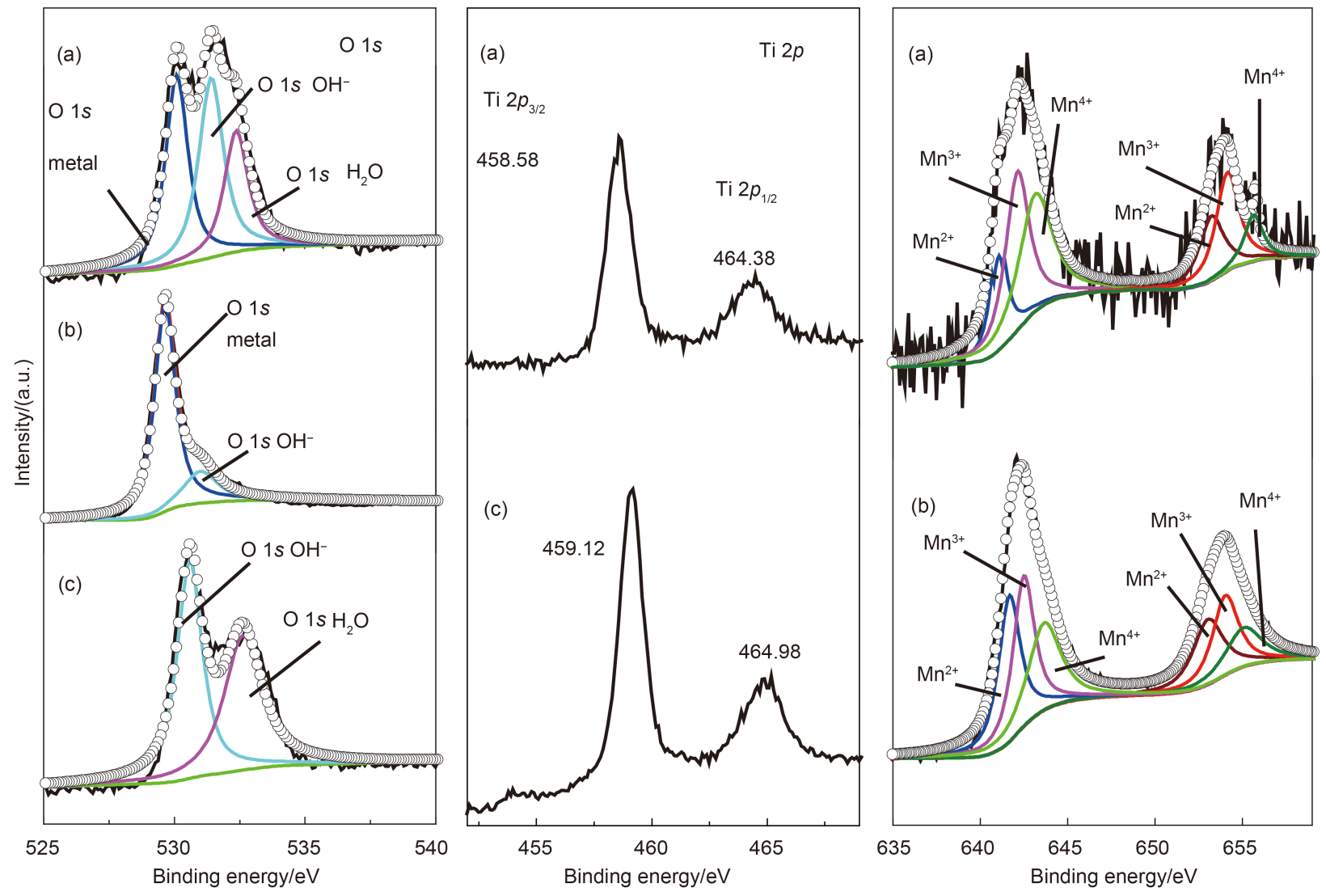

图6 $\alpha-\mathrm{MnO}_{2} / \mathrm{Ti} 、 \alpha-\mathrm{MnO}_{2}$ 粉末以及 $\mathrm{Ti}$ 基膜的XPS能谱图

Fig.6 XPS spectra of $\alpha-\mathrm{MnO}_{2} / \mathrm{Ti}, \alpha-\mathrm{MnO}_{2}$ powder, and Ti-based film

(a) $450{ }^{\circ} \mathrm{C} \alpha-\mathrm{MnO}_{2} / \mathrm{Ti}$; (b) $450{ }^{\circ} \mathrm{C} \alpha-\mathrm{MnO}_{2}$ powder; (c) Ti matrix

由图6还可以看出, 基膜 $\mathrm{Ti} 2 p_{2 / 3}$ 在 $459.12 \mathrm{eV}$ 以及 $464.98 \mathrm{eV}$ 存在Ti的特征峰, 两者相差 $5.8 \mathrm{eV}$, 表明 $\mathrm{Ti}$ 元素以 +4 价存在, ${ }^{24,25}$ 而 $450{ }^{\circ} \mathrm{C}$ 焙烧所得的 $\alpha-\mathrm{MnO}_{2} /$ $\mathrm{Ti}$ 催化膜 $\mathrm{Ti} 2 p$ 轨道谱峰向低结合能方向位移约 0.54 $\mathrm{eV}$, 即 $\mathrm{Ti}$ 的价态降低, 表明 $\mathrm{Ti}$ 的外层电子云密度增 加, 内部电子的结合能降低. ${ }^{26}$ 也就是说, $\mathrm{MnO}_{2}$ 纳米 颗粒与 $\mathrm{Ti}$ 基膜之间存在明显的化学键合作用. 由图 5(a)和图6中 $\mathrm{Mn}$ 谱峰可知, 在 $\alpha-\mathrm{MnO}_{2}$ 纳米颗粒表面 存在少量的 $\mathrm{Mn}^{2+}$, 在 $\mathrm{MnO}_{x} / \mathrm{Ti}$ 催化膜表面 $\mathrm{Mn}^{2+}$ 所占比 重显著降低, 主要为 $\mathrm{Mn}^{3+} 、 \mathrm{Mn}^{4+}$. 表明随着焙烧温度 的提高, $\mathrm{Mn}^{2+}$ 的含量逐渐降低, $\mathrm{Mn}^{3+} 、 \mathrm{Mn}^{4+}$ 含量逐渐 增大. 结合 $\mathrm{Ti}$ 的谱峰拟合结果, 进一步表明 $\alpha-\mathrm{MnO}_{2}$ 纳米颗粒与基膜表面的钛氧化物在焙烧过程中逐 渐形成 $\mathrm{Mn}-\mathrm{O}-\mathrm{Ti}$ 化学键, 从而导致 $\mathrm{Mn}$ 的平均价态 升高, Ti的结合能降低. ${ }^{27}$

为了表征 $\mathrm{MnO}_{x} / \mathrm{Ti}$ 催化膜的电化学性能, 首先 采用循环伏安法对其进行测试, 结果如图7所示. 由
图7看出, 基体多孔钛膜由于表面钛的氧化物存在 一定的电容性能. ${ }^{28} 25^{\circ} \mathrm{C}$ 负载 $\mathrm{MnO}_{x}$ 之后, 由于 $\mathrm{MnO}_{x}$ 结晶度低, 且存在有机物残留, 电容较低. 随焙烧温 度升高, $\mathrm{MnO}_{x}$ 结晶度提高且有机物热分解完全, 催 化膜电容逐渐增加. $450{ }^{\circ} \mathrm{C}$ 焙烧所制备的 $\alpha-\mathrm{MnO}_{2} / \mathrm{Ti}$ 具有最高的电容性能. 这主要由两方面原因所致: 一方面是由于 $\alpha-\mathrm{MnO}_{2}$ 与基体之间存在键合作用; 另 一方面 $\alpha-\mathrm{MnO}_{2}$ 具有锰氧八面体相互连接成的双链 微观结构和较高的结晶度, 以及表面的不饱和氧空 位也是其电容性能较好的主要因素. ${ }^{29,30}$

此外, 进一步采用计时电流法对 $\mathrm{MnO}_{x} / \mathrm{Ti}$ 电催 化膜电子传递性能进行考察, 结果如图8所示. 由图 8 可知, 随着扫描的进行, 不管是原始 Ti膜还是 250 、 $350{ }^{\circ} \mathrm{C}$ 所制得的 $\mathrm{MnO}_{x} / \mathrm{Ti}$ 电催化膜, 在初始阶段均由 于电极表面双电层的形成使得电流密度快速下降, ${ }^{31}$ 尔后随着时间的延长逐渐稳定, 并最终达到平衡(线 1、3、4). 特别是 $450{ }^{\circ} \mathrm{C}$ 所制备的 $\alpha-\mathrm{MnO}_{x} / \mathrm{Ti}$ (线 5)催 化膜不但下降趋势平缓, 而且平衡电流密度 $(1.5 \mathrm{~mA}$. 


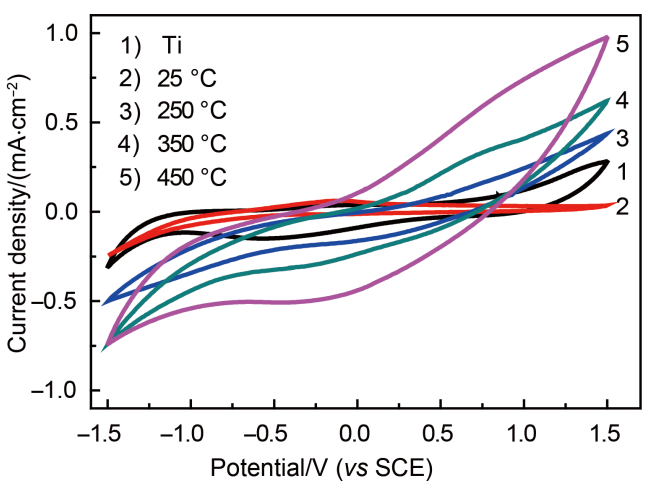

图7 不同焙烧温度 $\mathrm{MnO}_{\mathbf{x}} / \mathrm{Ti}$ 电催化膜循环伏安谱图

Fig.7 Cyclic voltammetry spectra of $\mathrm{MnO}_{x} / \mathrm{Ti}$ electrocatalytic membrane at different calcination temperatures

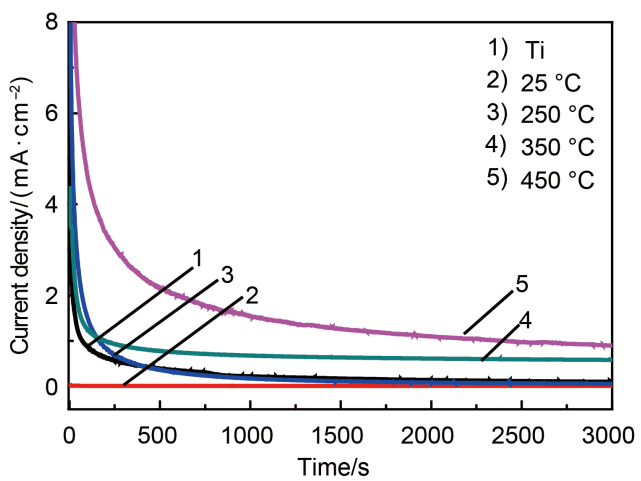

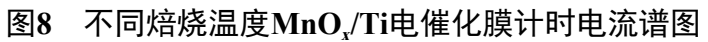

Fig.8 Chronoamperometry for $\mathrm{MnO}_{x} / \mathrm{Ti}$ electrocatalytic membrane at different calcination temperatures

$\mathrm{cm}^{-2}$ )明显高于其它膜. 这表明该膜电极不但传质效 率和电子传输速度更快, 而且稳定性更好. 而未经 过焙烧的催化膜则由于电流密度非常小而没有表 现出明显的下降趋势(线2).

\section{3 膜反应器催化氧化苯甲醇制备苯甲醛}

分别将原始多孔钛膜及不同焙烧温度下制备 的 $\mathrm{MnO}_{x} / \mathrm{Ti}$ 电催化膜作为阳极组装膜反应器进行苯 甲醇催化氧化制备苯甲醛. 反应溶液为 $50 \mathrm{mmol} \cdot \mathrm{L}^{-1}$ 苯甲醇水溶液, 以 $0.1 \mathrm{~mol} \cdot \mathrm{L}^{-1}$ 硝酸钠为电解质, 反应 温度为 $25^{\circ} \mathrm{C}$, 膜反应器电流密度为 $2 \mathrm{~mA} \cdot \mathrm{cm}^{-2}$, 停留 时间为 $15 \mathrm{~min}$, 催化结果如图9所示.

由图9可以看出, 采用基体钛膜时, 反应器苯甲 醇转化率为 $34.7 \%$, 苯甲醛选择性为 $75 \%$. 而采用未 经焙烧的 $\mathrm{MnO}_{x} / \mathrm{Ti}\left(25{ }^{\circ} \mathrm{C}\right)$ 膜时，由于其导电性能 差、电子传输效率低, 膜反应器苯甲醇转化率仅为 $10 \%$. 非常明显, 经过热处理后, 其催化活性逐渐提 高, 采用 $250 、 350$ 和 $450{ }^{\circ} \mathrm{C}$ 所制备 $\mathrm{MnO}_{\sqrt{ }} / \mathrm{Ti}$ 膜构建的

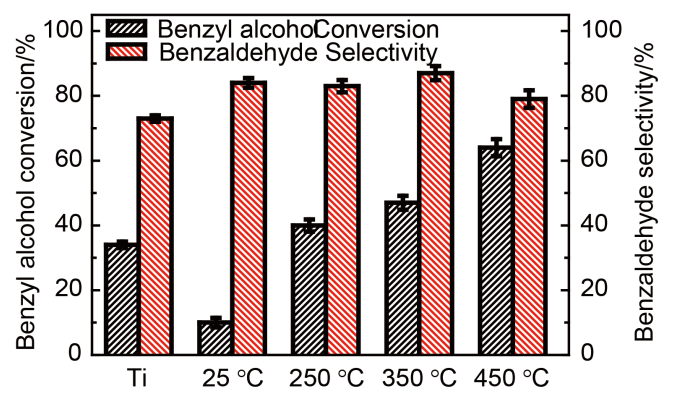

图9 $\mathrm{Ti}$ 基膜与不同焙烧温度 $\mathrm{MnO}_{\mathrm{x}} / \mathrm{Ti}$ 电催化膜氧化 性能比较

Fig.9 Comparison of the oxidation performance of $\mathrm{T} i$ matrix and $\mathrm{MnO}_{x} / \mathrm{Ti}$ membranes obtained at different calcination temperatures

膜反应器, 其苯甲醇转化率逐渐上升, 分别为 $40.4 \%$, $50.0 \%$ 和 $64 \%$. 同时, 苯甲醛选择性呈现先增加后降 低的趋势, 分别为 $83 \% 、 87 \% 、 79 \%$.

结合 $\mathrm{MnO}_{r} / \mathrm{Ti}$ 电催化膜的XRD、XPS、SEM、 $\mathrm{CV} 、 \mathrm{CA}$ 等表征结果, 可知在不同焙烧温度下热处 理可导致钛膜表面负载的 $\mathrm{MnO}_{x}$ 价态和相的转变, 特 别是经过 $450{ }^{\circ} \mathrm{C}$ 热处理后, $\mathrm{MnO}_{x}$ 含有更多的高价态 $\mathrm{Mn}^{4+}$ 以及可能存在更多的氧缺陷位和配位不饱和的 $\mathrm{Mn}$ 原子, 从而有利于电催化氧化反应的发生. 此外 随着焙烧温度的升高, 有机物热分解更彻底, 也就 是说 $\mathrm{MnO}_{x}$ 颗粒表面更加清洁, 更有利于苯甲醇反应 物分子的吸附, 从而提高苯甲醇氧化反应的转化率. 同时由于反应产物浓度的增加进而导致副反应发 生的几率增加, 因此在转化率提高的同时会导致选 择性有所下降。

\section{4 结 论}

采用乳液法将 $\mathrm{MnO}_{x}$ 纳米颗粒负载于管式微孔 钛膜, 制备出 $\mathrm{MnO}_{x} / \mathrm{Ti}$ 催化膜. 随着焙烧温度的升高, $\mathrm{MnO}_{x}$ 的晶型由 Birnessite- $\mathrm{MnO}_{2}$ 逐渐转变为 $\mathrm{K}_{0.27} \mathrm{MnO}_{2}$, 并经过 $\mathrm{Mn}_{3} \mathrm{O}_{4}$ 最终转变为 $\alpha-\mathrm{MnO}_{2}$, 且晶粒尺寸小于 $30 \mathrm{~nm} .450{ }^{\circ} \mathrm{C}$ 焙烧所制备的 $\alpha-\mathrm{MnO}_{2} / \mathrm{Ti}$ 催化膜由于 $\alpha-\mathrm{MnO}_{2}$ 表面含有较多的不饱和配位的锰原子和氧 缺陷位, 且与基体存在键合作用, 从而大大提高了 电催化膜的电化学性能和催化活性. 以 $\alpha-\mathrm{MnO}_{2} / \mathrm{Ti}$ 为 阳极构建电催化膜反应器, 在反应温度为 $25^{\circ} \mathrm{C}, 50$ $\mathrm{mmol} \cdot \mathrm{L}^{-1}$ 苯甲醇水溶液, 电流密度为 $2 \mathrm{~mA} \cdot \mathrm{cm}^{-2}$, 停 留时间为 $15 \mathrm{~min}$ 的条件下, 其苯甲醇转化率达 $64 \%$, 苯甲醛选择性为 $79 \%$. 


\section{References}

(1) Gelalcha, F. G. Chemical Reviews 2007, 107 (7), 3338. doi: $10.1021 / \mathrm{cr} 0505223$

(2) Dan, I. E.; Jennifer K. E.; Philip, L.; Benjamin, S.; Albert, F. C.; Andrew, A. H.; Masashi, W.; Christopher, J. K.; David, W. K.; Graham, J. H. Science 2006, 311, 5759.

(3) Opembe, N. N.; Guild, C.; King Ondu, C.; Nelson, N. C.; Slowing, I. I.; Suib, S. L. Industrial \& Engineering Chemistry Research 2014, 53 (49), 19044. doi: 10.1021/ie5024639

(4) Rezaei, S. E.; Zonoz, F. M.; Estiri, M.; Tayebee, R. Industrial \& Engineering Chemistry Research 2011, 50 (4), 1837. doi: 10.1021/ie101641t

(5) Parlett, C. M. A.; Bruce, D. W.; Hondow, N. S.; Lee, A. F.; Wilson, K. ACS Catalysis 2011, 1 (6), 636. doi: 10.1021/ cs200145n

(6) Mallat, T.; Baiker, A. Chemical Reviews 2004, 104 (6), 3037. doi: $10.1021 / \mathrm{cr} 0200116$

(7) Guo, Z.; Liu, B.; Zhang, Q.; Deng, W.; Wang, Y.; Yang, Y. Chemical Society Reviews 2014, 43 (10), 3480. doi: 10.1039/ c3cs60282f

(8) Camilla, P. F. C. Green Chem. 2012, 14, 547. doi: 10.1039/ c2gc16344f

(9) Choudhary, V. R.; Chaudhari, P. A.; Narkhede, V. S. Catalysis Communications 2003, 4 (4), 171.

(10) Su, Y.; Wang, L.; Liu, Y.; Cao, Y.; He, H.; Fan, K. Catalysis Communications 2007, 8 (12), 2181.

(11) Li, J.; Wang, H.; Li, J. X.; Guan, Q. Q.; Yang, Y. Membrane Science and Technology 2013, 33 (6), 64. [李 娇, 王 虹, 李建 新, 管崎崎, 杨 阳. 膜科学与技术, 2013, 33 (6), 64.]

(12) Li, J.; Li, J.; Wang, H.; Cheng, B.; He, B.; Yan, F.; Yang, Y.; Guo, W.; Ngo, H. H. Chemical Communications 2013, 49 (40), 4501. doi: 10.1039/c3cc41181h

(13) Wang, H.; Wang, H.; Li, J.; Bin, D.; Yin, Z.; Kang, J.; He, B. Electrochimica Acta 2014, 123, 33. doi: 10.1016/j.electacta. 2014.01. 016

(14) Bin, D.; Wang, H.; Li, J.; Wang, H.; Yin, Z.; Kang, J.; He, B.; Li, Z. Electrochimica Acta 2014, 130, 170. doi: 10.1016/j. electacta.2014.02. 128

(15) Fang, X.; Yin, Z.; Wang, H.; Li, J.; Liang, X.; Kang, J.; He, B. Journal of Catalysis 2015, 329, 187. doi: 10.1016/j.jcat. 2015.05.004
(16) Xiao, W.; Wang, D.; Lou, X. W. The Journal of Physical Chemistry C 2010, 114 (3), 1694. doi: 10.1021/jp909386d

(17) Chen, H.; He, J.; Zhang, C.; He, H. The Journal of Physical Chemistry C 2007, 111 (49), 18033.

(18) Lee, J. W.; Hall, A. S.; Kim, J.; Mallouk, T. E. Chemistry of Materials 2012, 24 (6), 1158. doi: $10.1021 / \mathrm{cm} 203697 \mathrm{w}$

(19) Devaraj, S.; Munichandraiah, N. The Journal of Physical Chemistry C 2008, 112 (11), 4406. doi: 10.1016/j.orgel. 2008.12.008

(20) Nesbitt, H. W. B. The American Mineralogist 1998, 83 (3-4), 305.

(21) Beyreuther, E.; Grafstr, M. S.; Eng, L. M. Physical Review B 2006, 73 (15), 155425. doi: 10.1103/PhysRevB.73.155425

(22) Kang, J.; Hirata, A.; Qiu, H. J.; Chen, L.; Ge, X.; Fujita, T.; Chen, M. Advanced Materials 2014, 26 (2), 269. doi: 10.1002/adma.201302975

(23) Dai, Y.; Li, J. H.; Peng, Y.; Tang, X. F. Acta Phys.-Chim. Sin. 2012, 7, 1771. [戴 韵, 李俊华, 彭 悦, 唐幸福. 物理化学学 报, 2012, 7, 1771.]. doi: 10.3866/PKU.WHXB201204175

(24) Crist, B. V. Handbook of Monochromatic XPS Spectra, Volume 1: The Elements of Native Oxides; Wiley \& Sons; Chichester, 2000; pp 515-519.

(25) Chen, Q. Y.; Tong, H. X.; Yin, Z. L.; Hu, H. P.; Li, J.; Liu, L. L. Acta Phys. -Chim. Sin. 2007, 12, 1917. [陈启元, 童海霞, 尹周 澜, 胡慧萍, 李 洁, 刘亮亮. 物理化学学报, 2007, 12, 1917.] doi: 10.3866/PKU.WHXB20071216

(26) Huang, Y.; Li, H.; Balogun, M.; Liu, W.; Tong, Y.; Lu, X.; Ji, H. ACS Applied Materials \& Interfaces 2014, 6 (24), 22920. doi: $10.1021 / \mathrm{am} 507641 \mathrm{k}$

(27) Zhang, X. J.; Liu, Q. J.; Deng, S. G.; Chen, J.; Gao, P. Acta Phys. Sin. 2011, 08, 567. [张学军, 柳清菊, 邓曙光, 陈 娟, 高 攀. 物理学报, 2011, 08, 567.]

(28) Lu, X.; Wang, G.; Zhai, T.; Yu, M.; Gan, J.; Tong, Y.; Li, Y. Nano Letters 2012, 12 (3), 1690.

(29) Zhang, Y.; Sun, C.; Lu, P.; Li, K.; Song, S.; Xue, D. CrystEngComm 2012, 14 (18), 5892. doi: 10.1039/c2ce25610j

(30) Cheng, F.; Su, Y.; Liang, J.; Tao, Z.; Chen, J. Chemistry of Materials 2010, 22 (3), 898. doi: 10.1021/cm901698s

(31) Dupont, M.; Hollenkamp, A. F.; Donne, S. W. Electrochimica Acta 2013, 104, 140. doi: 10.1016/j.electacta.2013.04.007 